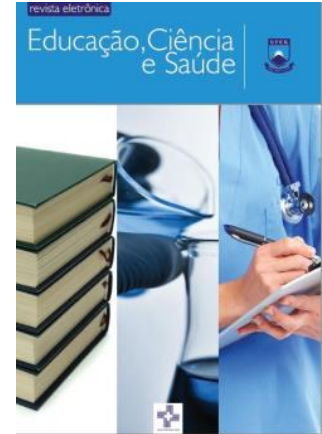

EDUCAÇÃO CIÊNCIA E SAÚDE

http://dx.doi.org/10.20438/ecs.v8i1.372

\title{
CONSTRUÇÃO DE CARTILHA EDUCATIVA SOBRE PRIMEIROS SOCORROS PARA PAIS E CUIDADORES DE CRIANÇAS: RELATO DE EXPERIÊNCIA
}

\author{
Glaycielle Fernandes de Oliveira ${ }^{1}$, Francisco de Assis Coutinho Pontes Júnior², \\ Maria Eduarda Camilo Damião ${ }^{3}$, Karen Lúcia de Freitas Moreira ${ }^{4}$, Stênio Melo \\ Lins da Costa ${ }^{4}$, Isolda Maria Barros Torquato ${ }^{4}$
}

${ }^{1}$ Acadêmica de fisioterapia, Universidade Federal da Paraíba, João Pessoa-PB, Brasil.

${ }^{2}$ Fisioterapeuta, Hospital Universitário Lauro Wanderley, João Pessoa, PB, Brasil.

${ }^{3}$ Fisioterapeuta, Fundação Centro Integrado de Apoio à Pessoa com Deficiência, João Pessoa, PB, Brasil.

${ }^{4}$ Fisioterapeuta, Departamento de Fisioterapia, Universidade Federal da Paraíba, João Pessoa, PB, Brasil.

E-mail para correspondência: isoldaufcg@gmail.com

\begin{abstract}
Resumo
Relatar a experiência sobre a produção e divulgação de uma tecnologia, em forma de cartilha educativa, acerca dos primeiros socorros nas principais situações de emergência pré-hospitalar com crianças. Estudo descritivo, do tipo relato de experiência sobre a construção de uma cartilha, tendo como públicoalvo pais, cuidadores e crianças assistidas na clínica escola infantil de fisioterapia da Universidade Federal da Paraíba. A elaboração foi realizada por estudante concluinte de fisioterapia, entre junho e outubro de 2020 e envolveu as seguintes etapas: (1) levantamento bibliográfico a partir da revisão da literatura; (2) elaboração do conteúdo; (3) criação das ilustrações por design gráfico; e (4) diagramação da cartilha. A construção da cartilha possibilitou a integração entre as pesquisadoras e o público alvo. O produto foi amplamente divulgado e houve retorno positivo das orientacões passadas de forma clara, objetiva e acessível. Os leitores relataram melhora do conhecimento sobre o tema de primeiros socorros para crianças. A experiência oportunizou a disseminação de informações seguras, com fontes confiáveis e embasamento científico, além da integração das pesquisadoras à comunidade. Ampliou-se o vínculo extramuros da universidade, fator imprescindível para atuação junto à população.
\end{abstract}

Palavras-chave: Educação em Saúde. Primeiros Socorros. Saúde da Criança. 


\begin{abstract}
Report the experience about the production and dissemination of a technology, in the form of an educational booklet, about first aid in the main pre-hospital emergency situations with children. Descriptive study, like an experience report on the construction of a booklet, with the target audience of parents, caregivers and children assisted in the clinic of the children's physiotherapy school at the Federal University of Paraíba. The preparation was carried out by a student completing physiotherapy, between June and October 2020 and involved the following steps: (1) bibliographic survey from the literature review; (2) elaboration of the content; (3) creation of illustrations by graphic design; and (4) layout of the booklet. The construction of the booklet enabled the integration between the researchers and the target audience. The product was widely publicized and there was a positive feedback from the guidelines given in a clear, objective and accessible way. Readers reported improved knowledge on the topic of first aid for children. The experience provided the opportunity for the dissemination of secure information, with reliable sources and scientific basis, in addition to the integration of the researchers into the community. The link outside the university was expanded, an essential factor for action with the population.
\end{abstract}

Keywords: Health Education. First Aid. Child Health.

\title{
1 Introdução
}

Os acidentes são definidos, culturalmente, como ocorrências inevitáveis, caracterizados como causas externas provenientes de um tipo de energia dinâmica, térmica ou química de um corpo a outro ocasionando danos e até o óbito (CORDEIRO et al., 2017).

As ocorrências de acidentes por causas externas constituíram-se nos últimos anos como a principal causa de mortalidade para a faixa etária de 12 meses aos 39 anos. Conforme a Organização Mundial de Saúde (OMS), aproximadamente 5,1 milhões de mortes são atribuídas às causas externas, representando 9\% da mortalidade mundial (BATISTA et al., 2018). Anualmente mais de 950.000 crianças e adolescentes perecem devido a acidentes causados por fatores externos (BELELA-ANACLETO; MANDETTA, 2016).

No Brasil, esses eventos correspondem a $3^{\text {a }}$ causa de morte em crianças entre zero e nove anos de idade. Em 2015, dados do Ministério da Saúde apontaram registros de 117 mil hospitalizações por acidentes em menores de 14 anos, sendo 36\% por acidentes de trânsito, 24\% por afogamento, $21 \%$ por sufocação, $6 \%$ por queimaduras e quedas, $1 \%$ intoxicações e 7\% estavam relacionados a outros tipos de acidentes (CRIANÇA SEGURA, 2015). 
Estudo revela relação entre a ocorrência dos acidentes na infância com as características de gênero, sendo mais prevalentes no sexo masculino, idade da criança, desenvolvimento neuropsicomotor e fatores predisponentes como os socioeconômicos (renda familiar e nível de escolaridade materna baixos); ambientais (riscos físicos, químicos e biológicos) e de vulnerabilidade (falta de afeto, violência urbana, desigualdades sociais, estresses, estado de pobreza, dentre outros) (MARTINS, 2015).

Considerando-se a elevada frequência desses tipos de acidentes, especialmente no contexto domiciliar, os quais influenciam diretamente na elevação epidemiológica da morbimortalidade em crianças por causas externas, faz-se necessário uma melhor abrangência do tema visando a elaboração de estratégias para reduzir esse problema de saúde pública (BATISTA et al., 2018).

Neste sentido, uma das ações fundamentais para a prevenção de acidentes domésticos assim como a adoção de medidas corretas de primeiros socorros envolve a orientação familiar por meio de ações educativas em saúde (PEREIRA et al., 2015).

A educação popular, nessa perspectiva, constitui-se como um instrumento facilitador para assistência qualificada, pois possibilita ressignificação de novos saberes, hábitos e práticas em saúde adequadas ao público envolvido. Entretanto, para isso, faz-se necessário que o processo de ensino-aprendizagem seja estabelecido por meio da escuta ativa e do estabelecimento de uma relação dialógica entre educando/família e o educador/profissional (AMARAL; MONTRONE, 2015).

Apesar de ser um tema relevante, o ensino de primeiros socorros à população ainda é pouco difundido no país junto ao público leigo, prevalecendo nesses, na maioria das vezes, o impulso da solidariedade em situações de urgência e emergência, o que pode acarretar em danos irreparáveis as vítimas (PEREIRA et al., 2015).

Desse modo, considerando a importância em orientar pais e cuidadores de crianças sobre as técnicas de primeiros socorros para que todos saibam como proceder em situações de emergência, justifica-se a necessidade da elaboração de materiais instrucionais para o processo de educação popular em saúde. 
O conhecimento e treinamento de técnicas básicas de primeiros socorros são indispensáveis para a sobrevida de vítimas em situações de emergências pré-hospitalar, até a chegada de serviço especializado (SILVA et al., 2020). Segundo estudo, a morbimortalidade pode ser reduzida em até $7,5 \%$ caso a primeira assistência seja prestada adequadamente por pessoas treinadas (OLIVEIRA et al., 2014).

Neste sentido, pode tornar-se valioso o preparo de adultos, jovens e inclusive de crianças para lidarem com situações, muitas vezes, inesperadas de acidentes por causas externas (GALINDO NETO et al., 2017).

Acredita-se que a capacitação da população leiga contribuirá com o trabalho de educação em saúde, desenvolvido por profissionais da área, e vêm ao encontro com a iniciativa da Política Nacional de Redução da Morbimortalidade por Acidentes e Violências do Ministério da Saúde (FERREIRA et al., 2017).

Cada vez mais se constata o uso de tecnologias educacionais, a exemplo de softwares, hipermídia, jogos, álbum seriado e cartilhas educativas como recursos voltados à promoção da saúde utilizados para esclarecer dúvidas e reforçar as orientações verbalizadas aos pais e familiares sobre temas inerentes a saúde da criança (ARAÚJO, 2016).

Desse modo, a disponibilidade de materiais educativos, a exemplo de cartilhas, pode constituir-se como um importante recurso didático para facilitar o entendimento das informações por parte do público que se deseja alcançar, conforme revela estudo desenvolvido com mães de crianças menores de dois anos, onde a utilização desse recurso se mostrou efetivo na ampliação de conhecimento e aplicação das orientações em domicílio (TORQUATO et al., 2019). Ademais, as cartilhas educativas podem auxiliar nas tomadas de decisões do cotidiano frente as situações que atribuam dúvidas (CASTRO; LIMA JÚNIOR, 2014).

Dessa forma, considerando-se a cartilha educativa como um instrumento relevante para propagar informações entre pais e cuidadores de crianças sobre condutas corretas em situações de emergência pré-hospitalar, o presente estudo teve como objetivo relatar a experiência sobre a produção e divulgação de uma tecnologia, em forma de cartilha educativa, acerca dos primeiros socorros nas principais situações de emergência pré-hospitalar com crianças. 


\section{Metodologia}

Trata-se de um relato de experiência com o objetivo de apresentar a produção e divulgação de uma cartilha educativa para orientar pais e cuidadores de crianças assistidas em serviço de fisioterapia infantil da Universidade Federal da Paraíba (UFPB) sobre as medidas de primeiros socorros em situações de emergência pré-hospitalar.

A cartilha é produto de um trabalho de conclusão de curso (TCC) elaborada considerando a demanda do público mencionado, a partir de um levantamento prévio sobre conhecimento de cuidadores acerca dos primeiros socorros em crianças. Desse modo, identificadas às fragilidades e as necessidades do grupo iniciou-se o processo de sistematização e elaboração do material educativo pelos pesquisadores, para possibilitar informações sobre como atuar frente às situações de emergência com crianças.

A construção da cartilha ocorreu entre junho e outubro de 2020, respeitando as seguintes etapas: levantamento bibliográfico; seleção do conteúdo; criação das ilustrações por design gráfico mediante orientação dos pesquisadores e diagramação da cartilha.

Para embasar a elaboração textual da cartilha educativa foram utilizados artigos científicos, livros, Guidelines e materiais educativos sobre primeiros socorros disponibilizados em site do Ministério da Saúde (MS), da Organização Mundial da Saúde (OMS) e da Sociedade Brasileira de Pediatria (SBP), por se tratarem de fontes atuais e confiáveis acerca da temática abordada.

Os conteúdos abordados na cartilha foram divididos em tópicos, a saber: cuidados quanto à segurança do local para realização dos primeiros socorros, acionamento do serviço de atendimento pré-hospitalar e os tópicos específicos para os principais agravos na criança (pancada e fratura, cortes e hemorragias, queimadura, febre e crise convulsiva, picadas de animais peçonhentos, parada cardíaca e corpos estranhos em orifícios).

Salienta-se que houve a adequação da linguagem científica, tornando-a mais apropriada ao público alvo.

Finalizada a construção, iniciou-se a divulgação on-line e o encaminhamento da cartilha para os pais e cuidadores das crianças em tele monitoramento e tele atendimento no serviço de fisioterapia da UFPB. 


\section{Resultados e Discussão}

O material tecnológico intitulado "Primeiros socorros: cartilha educativa para pais e cuidadores de crianças" foi produzida totalizando 21 páginas, divididas em sessões, as quais foram organizadas a fim promover linearidade e aprofundamento pelo leitor sobre o conteúdo abordado. Os temas escolhidos basearam-se nas principais situações de emergências ocorridas no cotidiano de crianças, conforme apontado pela literatura (ALBUQUERQUE et al., 2015; BATISTA et al., 2018). Foram eles: 1-Apresentação 2-Sumário 3-Primeiros socorros: o que são? 4-Quais os cuidados quanto a segurança do local para realização dos primeiros socorros? 5 -Como acionar o serviço de atendimento pré-hospitalar? 6-Primeiros socorros nas contusões e fraturas 7-Primeiros socorros nos cortes e hemorragias; 8-Primeiros socorros nas queimaduras 9Primeiros socorros na febre e crise convulsiva 10-Primeiros socorros nas picadas de animais peçonhentos 11-Primeiros socorros nos corpos estranhos em orifícios corporais 12-Primeiros socorros na parada cardíaca 13Referências 14-Autores.

A produção da cartilha educativa surgiu a partir das demandas dos pais e responsáveis das crianças identificadas pelos profissionais atuantes no serviço de fisioterapia da clínica infantil da UFPB, ou seja, a sua construção buscou atender as necessidades apontadas pela comunidade. Corroborando, estudo desenvolvido na periferia do município de Maceió (AL), o qual também utilizou a cartilha educativa como meio de promover a educação em saúde em população vulnerável (SILVA et al., 2020).

A utilização de tecnologias educativas de boa qualidade, a exemplo de cartilhas, construídas a partir de evidências científicas, podem constituir-se como uma estratégia eficaz para facilitar a compreensão das informações por parte do público-alvo, especialmente no contexto pandêmico atual da COVID19 em que os pais e cuidadores estão restritos nos seus domicílios (GALINDO NETO et al., 2017). Contudo, é essencial que se considere, durante a elaboração do material educativo, o nível de escolaridade a quem se destina, de maneira que a linguagem seja clara e objetiva para quem o lê (SANCHEZ; LEMOS; VERÍSSIMO, 2017). 
Nesse contexto, buscou-se elaborar uma cartilha com embasamento científico, porém, com linguagem acessível ao público alvo, a fim de facilitar o acesso às informações corretas e estimular mudanças de atitudes e comportamentos corretos em situações emergenciais com crianças.

A construção da cartilha foi um desafio para os pesquisadores, pois além da adaptação da linguagem para os leitores leigos houve a necessidade do desenvolvimento de ilustrações por um design gráfico, o qual os criou conforme as orientações e necessidades dos pesquisadores.

Ressalta-se que as ilustrações foram desenvolvidas para cada sessão mencionada na metodologia de maneira que, atreladas ao texto pudessem dar clareza e linearidade à obtenção de conhecimento pelos leitores. Somente após análise criteriosa das pesquisadoras, seguia-se com a aprovação ou não dos desenhos, conforme atendessem as descrições apresentadas no texto para evitar eventuais conflitos de entendimento ao leitor.

Conforme estudo, o uso de cartilhas utilizadas para fins educação em saúde pode constituir-se como um recurso complementar disponível aos sujeitos aprendizes, ajudando-os nas tomadas de decisões frente e a situações de emergência infantis (REBERTE; HOGA; GOMES, 2012).

Nesse contexto, considerando-se a elevada frequência de acidentes na faixa etária infantil, especialmente no âmbito domiciliar, percebeu-se a importância em disponibilizar ao público alvo um material gratuito, de fácil compreensão e acesso para que pudessem melhorar os conhecimentos e atitudes frente a situações de acidentes e emergências com crianças.

Pesquisadores relatam que a elaboração e oferta de materiais educativos contribuem para maior conhecimento e transformação social, especialmente entre o público de maior vulnerabilidade social (SILVA et al., 2020).

Nesse ínterim, acredita-se que, o processo de construção da cartilha possibilitou não apenas a disseminação do conhecimento ao público leigo como também a integração entre a discente e a pesquisadora responsável e o aprofundamento técnico-científico para a área temática.

A divulgação da cartilha ocorreu de forma on-line e gratuita, via mídia social, para os pais e cuidadores das crianças usuárias do serviço de 
fisioterapia. O reconhecimento sobre a importância do material produzido pelo público leitor a quem se destinou a cartilha foi imediato e positivo.

Ressalta-se que os pais e responsáveis elogiaram a iniciativa, no qual a cartilha permitiu acesso a informação, ampliação do conhecimento, troca de experiências entre estes e os pesquisadores envolvidos, como também a eliminação de dúvidas existentes sobre o assunto por parte dos pais e cuidadores, ou seja, foi possível o estabelecimento de um diálogo horizontal entre todos os envolvidos.

Dessa forma, foi possível inferir assim como no estudo de Torquato et al. (2019), que a educação popular em saúde é uma estratégia fundamental para assistência qualificada, possibilitando ressignificação de novos saberes, hábitos e práticas em saúde.

Contudo, apesar da importância do estudo, é preciso ressaltar a limitação da pesquisa, a qual esteve relacionada à impossibilidade de contato físico entre os participantes do grupo para promover intervenção educativa e treinamento das manobras e do conteúdo abordado, o que pode ter comprometido o conhecimento e o estabelecimento de diálogo ampliado entre a discente, a pesquisadora, os pais e os cuidadores.

\section{Conclusão}

A elaboração da tecnologia oportunizou a aproximação e o aprofundamento com o tema proposto, possibilitando a construção de uma experiência relevante para a estudante e a docente quanto à idealização e criação do material educativo. Além disso, disseminou informações com embasamento científico para melhor esclarecer aos pais e responsáveis de crianças sobre as formas corretas de cuidado nos primeiros socorros em situações de emergências.

Considerando a escassez de materiais educativos sobre o tema para o público leigo, sendo a cartilha elaborada com linguagem clara e com 0 propósito de melhorar o conhecimento, percebeu-se a boa aceitação e utilização pelos pais e responsáveis, refletindo a importância da educação popular no processo de ensino-aprendizagem no âmbito da saúde.

Indubitavelmente, a cartilha é mais um suporte para os pais e cuidadores de crianças, para que eles aprofundem informações, superem dúvidas e dificuldades que permeiam ações básicas de primeiros socorros na infância. 
Desse modo, pensando em ampliar o uso da cartilha para outros grupos fora do contexto da UFPB, pretende-se realizar brevemente um processo de validação da cartilha com juízes para verificar a cientificidade do material e utilizá-la em pesquisas futuras de caráter interventivo, sendo possível avaliar sua eficácia e consolidando novas evidências.

A sua validação representará um passo importante para que se busquem recursos junto a UFPB ou as entidades de fomento para sua publicação na versão impressa ou em formato e-book. Assim, as instituições públicas de saúde e todos àqueles que tenham interesse em ampliar 0 conhecimento sobre o assunto possam ter 0 acesso à cartilha de forma gratuita.

\section{Referências}

ALBUQUERQUE, A. M. de et al. Salvando vidas: avaliando o conhecimento de adolescentes de uma escola pública sobre primeiros socorros. Rev enferm UFPE on line., v. 9, n. 1, p. 32-8, 2015.

AMARAL, D. M. do; MONTRONE, Aida Victoria Garcia. Educação popular: Metodologia de pesquisa como processo educativo. Revista da FAEEBA: Educação e Contemporaneidade, Salvador, v. 24, n. 43, p. 75-88, 2015.

ARAÚJO, M. M. de. Construção e validação de cartilha educativa para orientação dos pais sobre asma na infância. 2016. 115f. (Mestre em Enfermagem) Universidade Federal do Ceará, Fortaleza, 2016.

BATISTA, J.; BARRETO, M. S.; MERINO, M. F. G. L; et al. Perfil Epidemiológico da Mortalidade por Causas Externas Entre Beneficiários de Planos de Saúde no Brasil. Revista de Enfermagem do Centro-Oeste Mineiro. Revista de Enfermagem do Centro-Oeste Mineiro, v. 8; e1870, p. 1-12, 2018.

BELELA-ANACLETO, S. C. A; MANDETTA, M. A. Prevenção de acidentes na infância: uma convocação da "Sociedade Brasileira de Enfermeiros Pediatras". Acta Paulista de Enfermagem, v. 29, n.5, p. 1-2, 2016.

CASTRO, A. N. P.; LIMA JUNIOR, E. M. Desenvolvimento e validação de cartilha para pacientes vítimas de queimaduras. Rev Bras Queimaduras, v.13, n.2, p.103-113, 2014.

CORDEIRO, R.; LUZ, V. G.; HENNINGTON, E. A.; MARTINS, A. C. A.; TÓFOLI, L. F. A violência urbana é a maior causa de acidente de trabalho fatal no Brasil. Rev Saude Publica, v. 51, n. 123, p.1-12, 2017.

CRIANÇA SEGURA BRASIL. Relatório institucional 2015/2016. São Paulo: 2015 
FERRAI L. R. The pediatric airway: anatomy, challenges, and solutions. Em: Mason KP, editor. Pediatric sedation outside of the operating room. 2nd ed. New York: Springer; 2015. p. 95-109.

FERREIRA, M. das G. N.; ALVES, S. R. P.; SOUTO, C. G. V. de; VIRGÍNIO, N. de A.; SILVA JÚNIOR, J. N. de B.; SANTOS, A. F. dos. O LEIGO EM PRIMEIROS SOCORROS: Uma revisão integrativa. Revista de Ciências da Saúde: Nova Esperança, João Pessoa, v. 15, n. 3, 2017.

GALINDO NETO, N. M. Tecnologia educativa para professores sobre primeiros socorros: Construção e validação. 2015. 138 f. (Mestre em Enfermagem) Universidade Federal de Pernambuco, 2015.

MARTINS, H. S.; BRANDÃO NETO, R. A.; VELASCO, I. T. Emergências clínicas: abordagem prática. 11. ed. São Paulo: Manole, 2015.

OLIVEIRA, M. A. J; SILVA C. J. J.; TOLEDO E. M. O Conhecimento em ProntoSocorrismo de Professores da Rede Municipal de Ensino do Ciclo I de Cruzeiro-SP. Rev. Educação, Cultura e Comunicação, ECCOM, v. 4, n. 7, 2014.

PEREIRA, K. C.; PAULINO, J. R.; SALTARELLI, R. M. F.; et al. A construção de conhecimentos sobre prevenção de acidentes e primeiros socorros por parte do público leigo. RECOM: Revista de Enfermagem do Centro Oeste Mineiro, v. 5, n. 1, p. 1478-85, 2015.

REBERTE, L. M.; HOGA, L. A. K.; GOMES, A. L. Z. G. O processo de construção de material educativo para a promoção da saúde da gestante. Rev. Latino-Am. Enfermagem, v. 20, n. 1, p. 1-8, 2012.

SANCHEZ, M. P. C.; LEMOS, R. A.; VERÍSSIMO, M. de L. R. Avaliação de materiais educativos para o cuidado e a promoção do desenvolvimento de crianças nascidas prematuras. Rev. Soc. Bras. Enferm. Ped., v. 17, n. 2, p. 76-82, 2017.

SILVA, R. de C. R. da; RAIMUNDO, A. C. de L.; SANTOS, C. T. O. dos; VIEIRA, A. C. S. Construção de cartilha educativa sobre cuidados com crianças frente à pandemiacovid-19: relato de experiência. Rev baiana enferm, v. 34, e. 37173, p. 1-7, 2020.

TORQUATO, I. M. B.; COLLET, N.; FORTE, F. D. S.; FRANÇA, J. R. F. S.; SILVA, M. F. O. C.; REICHERT, A. P. S. Efetividade de uma intervenção com mães para a estimulação de crianças menores de dois anos. Rev. Latino-Am. Enfermagem, v. 27, n. e-3216. p. 1-10, 2019. 